\title{
Testicular endocrine activity is upregulated by D-aspartic acid in the green frog, Rana esculenta
}

\author{
F Raucci $^{1}$, L Assisi ${ }^{2}$, S D'Aniello ${ }^{3}$, P Spinelli $^{3}$, V Botte $^{2}$ and \\ M M Di Fiore ${ }^{1}$ \\ ${ }^{1}$ Department of Life Sciences, Second University of Naples, Via Vivaldi 43, 81100 Caserta, Italy \\ ${ }^{2}$ Department of Zoology, University of Naples, Via Mezzocannone 8, 80134 Naples, Italy \\ ${ }^{3}$ Department of Neurochemistry, Zoological Station of Naples, Villa Comunale, 80121 Naples, Italy \\ (Requests for offprints should be addressed to MM Di Fiore; Email: MariaM.DiFiore@unina2.it)
}

\begin{abstract}
This study investigated the involvement of D-aspartic acid (D-Asp) in testicular steroidogenesis of the green frog Rana esculenta and its effect on stimulation of thumb pad morphology and glandular activity, a typical testosteronedependent secondary sexual characteristic in this amphibian species. In the testis, D-Asp concentrations vary significantly during the reproductive cycle: they are low in pre- and post-reproductive periods, but reach peak levels in the reproductive period $(140-236 \mathrm{nmol} / \mathrm{g}$ wet tissue). Moreover, the concentrations of $\mathrm{D}-\mathrm{Asp}$ in the testis through the sexual cycle positively match the testosterone levels in the gonad and the plasma. The racemase activity evaluated during the cycle expresses its peak when D-Asp and testosterone levels are highest, that is, during the reproductive period, confirming the synthesis of D-Asp from L-Asp by an aspartate racemase. Short-term in vivo experiments consisting of a single injection of $\mathrm{D}$-Asp $(2 \cdot 0 \mu \mathrm{mol} / \mathrm{g}$ body weight $)$ demonstrated that this amino acid accumulates significantly in the testis, and after $3 \mathrm{~h}$ its uptake is coupled with a testosterone increase in both testis and plasma. Moreover, within $18 \mathrm{~h}$ of amino acid administration, the D-Asp concentration in the testis decreased along with the testosterone titer to prestimulation levels. Other amino acids (L-Asp, D-Glu and L-Glu) used instead of D-Asp were ineffective, confirming that the significant
\end{abstract}

increase in testicular testosterone was a specific feature of this amino acid. In long-term experiments, D-Asp had been administered chronically to frogs caught during the three phases of the reproductive cycle, inducing testosterone increase and $17 \beta$-estradiol decrease in the gonad during the pre- and post-reproductive period, and vice versa during the reproductive period.

The stimulatory effect of D-Asp on testosterone production by the testis is consistent with the stimulation of spermatogenesis and the maturation of thumb pads occurring in D-Asp-treated frogs. In these last animals, there was an increase of seminiferous ampoule area and a higher number of spermatids and sperm. Moreover, in spermatogonia I and II and in spermatocytes, a proliferating cell nuclear antigen (PCNA) intense immunopositivity was observed. In addition, the thumb pads of D-Asptreated frogs compared with controls showed a significantly thicker epithelial lining, a wider area of their glands with taller secretion cells, and more numerous, PASpositive-rich secretions. Finally, these results provide functional evidence for a biologic role of D-Asp in amphibian male steroidogenesis; therefore, this unusual amino acid could be considered a modulatory agent for reproductive processes.

Journal of Endocrinology (2004) 182, 365-376

\section{Introduction}

Since the discovery of significant amounts of D-aspartic acid (D-Asp) in several invertebrate organs (D'Aniello \& Giuditta 1977, 1978, D’Aniello et al. 1993a), studies have revealed its occurrence and physiological role(s) in vertebrate tissues. Most recent investigations have concerned the nervous and endocrine system of mammals, including humans (Dunlop et al. 1986, Fisher et al. 1991, Hashimoto et al. 1993, Hashimoto \& Oka 1997, D'Aniello et al. 1998a), where D-Asp is well represented. In the nervous

system, D-Asp is now accepted as one of the numerous agents involved in neurotransmission and neurosecretion (Schell et al. 1997); it is, in fact, localized in different nuclei of rat brain (Wang et al. 2002), particularly those engaged in neurosecretion (Schell et al. 1997, D'Aniello et al. 2000a, Wang et al. 2000, 2002).

D-Asp is also a regular component of endocrine glands, such as the pineal gland, hypothalamus, pituitary gland, adrenals, and gonads, where, according to experimental studies, it helps regulate hormone synthesis and/or release. The highest concentrations of D-Asp have been observed 
in the rat pineal gland (Imai et al. 1995, Lee et al. 1997), and here the amino acid modulates the secretion (Takigawa et al. 1998) and synthesis (Ishio et al. 1998) of melatonin in pinealocytes. Furthermore, D-Asp is thought to stimulate hormone production in rat hypothalamic nuclei, as well as hormone production and secretion in the rat adeno- (D'Aniello et al. 2000a,b) and neuro-hypophysis (Wang et al. 2000).

Outside the brain, D-Asp has been localized in the adrenal medulla (Lee et al. 2001), where it occurs at high concentrations (Schell et al. 1997), and in the testis (Sakai et al. 1998, D'Aniello et al. 1998b, Nagata et al. 1999a). As for the gonads, in the rat testis the amino acid is contained in spermatids (Sakai et al. 1998) and in both Leydig (D'Aniello et al. 1998b, Nagata et al. 1999a) and Sertoli (D'Aniello et al. 1998b) cells, and, as shown by in vivo and in vitro tests, it operates on these cells' steroidogenetic activities through either an indirect or a direct mechanism. The indirect mechanism follows D-Asp-induced growth hormone-releasing hormone $(\mathrm{GnRH})$ secretion by the hypothalamus, which entails luteinizing hormone (LH) release by the anterior pituitary (D'Aniello et al. 2000a). Gonadotropin, in turn, upregulates testosterone production by the Leydig cells. In rat testis, D-Asp acts directly on Leydig cells by enhancing the production of a specific mRNA and related protein, steroidogenic acute regulatory protein (StAR) (Nagata et al. 1999b), which is a key factor in gonadal steroidogenesis. Various observations assign a role for $\mathrm{D}-\mathrm{Asp}$ in the regulation of testosterone production. A direct D-Asp mechanism, on the other hand, finds support in the enhancement of testosterone production in testis tissue cultures to which the amino acid has been added (D'Aniello et al. 2000a).

Although most studies on D-Asp intervention in steroidogenesis have so far concerned laboratory mammals, we cannot rule out that these D-Asp properties are widespread in vertebrates, since they have been reported in females of the green frog Rana esculenta (Di Fiore et al. 1998) and of the lizard Podarcis s. sicula (Assisi et al. 2001). D-Asp enhances $17 \beta$-estradiol production by the ovarian follicles through upregulation of local aromatase activity. For D-Asp, this finding suggests a different target on sex steroid production machinery depending on the species and/or the sex; that is, the amino acid could operate differently in mammalian and nonmammalian species, and/or favor androgen production in males and estrogen production in females. There are no studies yet available on the presence of D-Asp and its effect on steroidogenesis in males of lower vertebrate species.

Therefore, to get more information on this interesting aspect of D-Asp functions in the vertebrate gonad, we investigated $\mathrm{D}$-Asp in the testis of the green frog, $R$. esculenta, and evaluated this amino acid's effect on sex hormone production (testosterone and $17 \beta$-estradiol) by the male gonad. Moreover, for functional evidence of a biologic role of $\mathrm{D}$-Asp in steroidogenesis, we considered two important androgen-dependent targets of significant physiological value: the testis and the thumb pad.

\section{Materials and Methods}

\section{Animals}

Adult males of the green frog, R. esculenta, weighing about $15 \mathrm{~g}$, were caught in marshes (Castelvolturno, Italy) during the three main phases of their reproductive cycle, namely, October (pre-reproductive period), FebruaryMarch (reproductive period) and July (post-reproductive period). Some animals were killed soon after capture, whereas others were transferred to the laboratory terrarium and maintained in a photothermal regime identical to the corresponding period of the year. Captive frogs were fed on mealworms and Drosophila fruit flies ad libitum. Mortality rates were low $(<8 \%)$. These frogs were used for experiments in vivo.

To evaluate the D-Asp and sex hormone content in the gonads and in the blood, five males, for each phase of their sexual cycle, were utilized soon after capture. They were anesthetized through immersion in a 1:1000 tap water solution of MS-222 (tricaine methylsulfonate, Sigma). Thereafter, blood was collected through the insertion of a heparinized glass capillary in the heart conus arteriosus; the blood samples were then centrifuged to obtain plasma, which was frozen in liquid nitrogen. At dissection, the reproductive stage was assessed by visually inspecting the gonads and secondary sexual characteristic (thumb pad). Thereafter, the testes and the thumb pads were removed and immediately frozen in liquid nitrogen or fixed by immersion in Bouin's fluid.

Experiments To determine the D-Asp effect on testicular steroid production, both short-term and long-term experiments were carried out.

In the first experiment (short-term), 40 frogs were distributed into two groups (20 animals each). Animals from the first group were injected i.p. with $2 \cdot 0 \mu \mathrm{mol}$ D-Asp (Sigma)/g body weight dissolved in $0.25 \mathrm{ml}$ amphibian saline. This dose was chosen on the basis of preliminary experimental tests. The animals of the second group received $0.25 \mathrm{ml}$ amphibian saline alone, and, therefore, were used as controls. Five injected frogs from each group were killed at set times within a period of $24 \mathrm{~h}(3,6,18$ and $24 \mathrm{~h}$ after the amino acid injection respectively). The frogs were dissected, and plasma and testes were obtained as reported above and stored in liquid nitrogen until use.

In the long-term experiments, frogs were sorted into groups, each formed by five animals. In a first experiment, carried out on post-reproductive animals (July), the animals were distributed into four groups and the treatments were as follows: frogs from groups $1,2,3$ and 4 received i.p. $2 \cdot 0 \mu \mathrm{mol} / \mathrm{g}$ body weight of different amino acids dissolved in $0.25 \mathrm{ml}$ of amphibian saline, every 2 days for 
2 weeks; that is, group 1 was injected with $\mathrm{D}$-Asp, group 2 with L-aspartate (L-Asp), group 3 with D-glutamate (D-Glu) and group 4 with L-glutamate (L-Glu). The amino acids were purchased from Sigma. The frogs of group 5 were injected as above, but with solvent alone (physiological saline), and used as controls. Two days after the last injection, the animals were killed and used as reported above, but, in addition, one testis and one thumb pad of each animal were rapidly fixed and processed for histology and immunohistochemistry as below.

A second long-term experiment was carried out on frogs caught in three periods of their sexual cycle, that is, pre-reproductive, reproductive and post-reproductive. The animals were sorted into two groups, and treated with D-Asp or amphibian saline alone respectively, as reported in the previous experiment. From these frogs, one testis and one thumb pad of each animal were rapidly fixed and processed as reported below.

The methods of capture and dissection and the captive rearing conditions were in accordance with Italian law (D. L.vo 116/92) and authorized by the appropriate Italian government administrative office (Servizio veterinario della A.S.L. 44, Prot. Vet. 22/95).

\section{Sex steroid assays in plasma and testis}

Sex steroid in the plasma was determined with enzyme immunoassay (EIA) kits (Biochem Immuno Systems, Bologna, Italy). The following limits of detection were observed: for testosterone, sensitivity was 6 pg (intra-assay variability $5 \cdot 6 \%$, interassay variability $9 \cdot 3 \%$ ); for $17 \beta$ estradiol, sensitivity was $6 \mathrm{pg}$ (intra-assay variability $5 \cdot 3 \%$, interassay variability $7 \cdot 5 \%$ ). The addition of D-Asp to the standard curve did not modify the assay sensitivity. Plasma samples (100-200 $\mu \mathrm{l})$ were vortexed with ethyl ether (1:10, $\mathrm{v} / \mathrm{v})$ for $5 \mathrm{~min}$ and centrifuged at $3000 \mathrm{~g}$ for $10 \mathrm{~min}$. The upper phase (ethyl ether) was transferred to a glass tube. Two extractions were performed. The pooled ether phases were left to evaporate on a hot plate at $40-50{ }^{\circ} \mathrm{C}$ under a hood. The residue was dissolved in a $0.5 \mathrm{ml}$ sodium phosphate buffer $0 \cdot 05 \mathrm{M}, \mathrm{pH} 7 \cdot 5$, containing BSA at a concentration of $10 \mathrm{mg} / \mathrm{ml}$, and then utilized for the assay.

Tissue samples (testis) were homogenized 1:5 (w/v) with distilled water. The homogenate was then mixed vigorously with ethyl ether $(1: 10 \mathrm{v} / \mathrm{v})$, and the ether phase was withdrawn after centrifugation at $3000 \boldsymbol{g}$ for $10 \mathrm{~min}$. Three extractions were performed. Sex steroid recovery was $85 \%$ from plasma, and $80 \%$ from tissues. Pooled ether extracts were dried and then utilized for the enzyme immunoassay as previously reported.

\section{Tissue preparation}

Tissue samples were homogenized with $0 \cdot 2 \mathrm{M}$ perchloric acid (PCA) in a 1:10 ratio and centrifuged at $30000 \boldsymbol{g}$ for $20 \mathrm{~min}$. The supernatant was loaded on a column of cation-exchange resin (AG 50W-X8 resin, hydrogen ionic form, 200-400 mesh, Bio-Rad), using a column of $1 \mathrm{~cm}$ diameter and an amount of resin corresponding to $1 \mathrm{ml}$ of settled resin for each $\mathrm{ml}$ of PCA supernatant. The resin was previously activated by treatment with $6 \mathrm{M} \mathrm{HCl}$ and then washed with 0.001 M HCl. Next, the sample was absorbed on the resin, and the column was washed with $10 \mathrm{ml} 0 \cdot 01 \mathrm{M} \mathrm{HCl}$ and then eluted with $3 \times$ volume resin of $4 \mathrm{M} \mathrm{NH} \mathrm{NH}_{4} \mathrm{OH}$. The ammonia eluted was dried by evaporation in small Petri dishes on a hot plate at $40-60{ }^{\circ} \mathrm{C}$ under a hood. The dry residue was dissolved in $2 \mathrm{ml}$ distilled water which contained free amino acids without salts, lipids and other organic compounds. However, this sample still contained pigments and molecules that can interfere with the enzymatic assay of $\mathrm{D}$-amino acids. Therefore, the sample was still-purified on a Octadecyl C-18 resin as follows. The sample was slowly passed through a Sep-pak C-18 cartridge containing 400-600 mg resin (the cartridge had been previously activated with methanol or acetonitrile and washed with distilled water). After the sample had passed through the cartridge, the cartridge was washed with $2 \mathrm{ml}$ distilled water, and all eluates were combined and concentrated to $200 \mu$ with a Savant centrifuge or left to evaporate in small Petri dishes at $40-50{ }^{\circ} \mathrm{C}$ under the hood.

Specific determination of $\mathrm{D}$-aspartic acid The content of D-Asp in the purified sample was determined specifically by the HPLC method associated with the use of D-aspartate oxidase (D-AspO) (EC 1.4.3.1.), as previously described (Di Fiore et al. 1998). This procedure is based on the chromatographic separation of D-Asp from other amino acids and through the oxidation of D-Asp with D-AspO (D'Aniello et al. 2000a). The purified D-AspO enzyme was obtained by overexpression and purified according to the described procedure (Negri et al. 1999).

A standard curve was obtained with D-Asp solutions of known concentrations (D'Aniello et al. 1993b, Tedeschi et al. 1994). Recovery studies were performed by adding D-Asp at different concentrations to a sample homogenate, and D-Asp was determined before and after addition of D-Asp. The percent (\%) recovery obtained was $94-108 \%$. The interassay coefficient of variation $(\mathrm{CV})$ was evaluated at several points along the standard curve in three different assays. The CV was $4 \cdot 5-8 \cdot 5 \%$ in the range $10-100 \mathrm{pmol}$ of D-Asp. The data were statistically analyzed by the STATVIEW program, version 4 (Abacus Concepts, Berkeley, CA, USA). This method allowed us to determine a minimum amount of $\mathrm{D}$-Asp corresponding to a $10 \mathrm{pmol} /$ assay mixture.

\section{Biosynthesis of $D$-Asp: racemase activity}

To verify whether D-Asp is biosynthesized from L-Asp by an aspartate racemase, we measured the racemase activity 
by evaluating the in vitro conversion rate of L-Asp into D-Asp. Testis and liver samples (five for each period) were homogenized $(1: 10 \mathrm{w} / \mathrm{v})$ in $0.05 \mathrm{M}$ sodium phosphate buffer, $\mathrm{pH} 7 \cdot 4$, containing $10 \mathrm{mM}$ of sodium EDTA and centrifuged at 30000 r.p.m. for $30 \mathrm{~min}$. The supernatants were dialyzed against 11 of the same buffer for $6 \mathrm{~h}$ followed by another dialysis cycle for $6 \mathrm{~h}$ against a new liter of the buffer. The dialyzed samples (without endogenous amino acids) were centrifuged as above, and the supernatants were used to measure racemase activity. The assay had two steps: step 1), conversion of L-Asp to D-Asp; step 2), determination of neosynthesized D-Asp.

The enzymatic reactions were as follows:

Racemase L-Aspartic acid $\longrightarrow$ D-aspartic acid

D-AspO D-Aspartic acid $+\mathrm{O}_{2}+\mathrm{H}_{2} \mathrm{O} \longrightarrow$ oxaloacetate + $\mathrm{H}_{2} \mathrm{O}_{2}+\mathrm{NH}_{3}$

Oxaloacetate $+2,4$-dinitrophenylhydrazine $=$ color complex (yellow)

Color complex (yellow) $+\mathrm{NaOH}=$ enhanced color complex (purple color)

Step 1 In microcentrifuge tubes, we mixed $50 \mu$ of the sample with $50 \mu \mathrm{l} 0.2 \mathrm{M} \mathrm{L}$-Asp and $50 \mu \mathrm{l} 1.0 \mathrm{M}$ citrate phosphate buffer at $\mathrm{pH}$ values between $4 \cdot 0$ to $8 \cdot 0$, and incubated the mixture for $120 \mathrm{~min}$ at $37^{\circ} \mathrm{C}$. After incubation, $50 \mu \mathrm{l} 1.0 \mathrm{M}$ PCA were added to stop the reaction. The samples were centrifuged at $15000 \mathrm{~g}$ for $5 \mathrm{~min}$, and the supernatants were brought to $\mathrm{pH} 8 \cdot 0-8 \cdot 5$ with $1 \cdot 0 \mathrm{M}$ $\mathrm{KOH}$. They were then cooled for about $30 \mathrm{~min}$ at $0{ }^{\circ} \mathrm{C}$ to render the potassium perchlorate from the reaction between PCA and $\mathrm{KOH}$ completely insoluble, and centrifuged as above.

Step 2 A volume of $100 \mu \mathrm{l}$ of neutralized sample from the first step was buffered to $\mathrm{pH} 8 \cdot 2$ by adding $50 \mu \mathrm{l} 1 \cdot 0 \mathrm{M}$ Tris $\mathrm{HCl}, \mathrm{pH} 8 \cdot 2$, and incubated with $5 \mu \mathrm{l}$ of purified $\mathrm{D}-$ AspO $(5 \mathrm{mg} / \mathrm{ml})$ for $30 \mathrm{~min}$ at $37^{\circ} \mathrm{C}$. After incubation, $30 \mu \mathrm{l} 2$,4-dinitrophenylhydrazine $(5.0 \mathrm{mM}$ in $\mathrm{HCl} 5 \mathrm{M})$ were added and mixed; after $10 \mathrm{~min}, 500 \mu \mathrm{l} \mathrm{NaOH} \mathrm{1.0} \mathrm{M}$ were added and mixed. After $5 \mathrm{~min}$, the assay mixtures were centrifuged in a table top centrifuge at 13000 r.p.m. for $5 \mathrm{~min}$, and the absorbance of the samples was read at $445 \mathrm{~nm}$ against the blank.

To determine the amount of D-Asp synthesized, a standard of D-Asp was performed, using $100 \mu \mathrm{l}$ D-Asp standard $(0.1 \mathrm{mM})$ instead of the sample, and a blank sample was performed under the same conditions, but without the D-AspO.

The results are expressed as enzymatic units/g tissue ( $\mathrm{U} / \mathrm{g}$ tissue). One unit is defined as the amount of the enzyme, contained in $1 \mathrm{~g}$ tissue, able to convert $1 \mathrm{nmol}$ of L-Asp to D-Asp under the above assay conditions.

In this study, in addition to L-Asp as substrate, other L-amino acids (L-Glu and L-Ala) and $\mathrm{NaCl}$ (as control) were also used to determine the specificity of the racemase substrate.

\section{Histology and histochemistry}

After the dissection, testes and thumb pads were rapidly immersed in Bouin's fluid. The histologic morphology of testes was studied in paraffin section $(5 \mu \mathrm{m})$ stained with hematoxylin and eosin (HE) and Mallory reactions. The histologic and histochemical properties of secretory granules of thumb pads were studied in paraffin section $(5 \mu \mathrm{m})$ stained with $\mathrm{HE}$ and Mallory reactions, periodic acid-Schiff (PAS), Alcian blue/PAS (AB/PAS), Alcian blue in 3\% acetic acid ( $\mathrm{pH} 2.5)$ and bromophenol blue, as described in Mazzi (1977).

PCNA immunocytochemistry To assess cell proliferation, nuclear protein proliferating cell nuclear antigen (PCNA) immunohistochemistry was performed by the procedure reported by Chieffi et al. (2000). Frog testes, rapidly removed and fixed in Bouin's fluid, were serially dehydrated in ethanol and cleared in xylene. For each paraffin-embedded sample, 4- $\mu \mathrm{m}$-thick serial sections, mounted on slides, were dewaxed in xylene and brought through ethanols to deionized distilled water. Ten sections per animal per month were examined. The endogenous peroxidases were quenched by incubation of sections in $0 \cdot 1 \%$ sodium azide with $0 \cdot 3 \%$ hydrogen peroxide for $30 \mathrm{~min}$ at $22^{\circ} \mathrm{C}$. The conventional avidin-biotin complex (ABC) procedure was used (Hsu et al. 1981). PCNA expression was tested by using the anti-PCNA antibodies at a dilution of 1:200. The peroxidase activity was developed with a filtered solution of $5 \mathrm{mg} \mathrm{3,3^{ \prime } -}$ diaminobenzidine tetrahydrochloride (Sigma) dissolved in $15 \mathrm{ml}$ Tris buffer $0.05 \mathrm{M}, \mathrm{pH} 7 \cdot 6$, and $0.03 \% \mathrm{H}_{2} \mathrm{O}_{2}$. Sections were mounted with a synthetic medium.

The following controls were performed: (1) omission of the primary antibody; (2) substitution of the primary antiserum with no immune serum (Dako Cytomation SpA, Milan, Italy) diluted 1:500 in blocking buffer. No immunostaining was observed after any of the control procedures.

Morphometry Five randomly chosen sections of testis and thumb pad for each frog from each experimental group (three per stage) were viewed by an image analyzer system. The following morphologic parameters were measured: the seminiferous ampoule area $\left(\mu \mathrm{m}^{2}\right)$ and number of spermatids and sperm for testes; the epithelial lining $(\mu \mathrm{m})$ of thumb pads, their gland areas $\left(\mu \mathrm{m}^{2}\right)$ and, for each gland, the height $(\mu \mathrm{m})$ and the number of secretory cells.

The morphometric analysis consisted of digitization of transverse sections viewed under a Nikon Eclipse E600 light microscope with an attached JVC TK-C1381 color photo camera connected to a Pentium II computer 


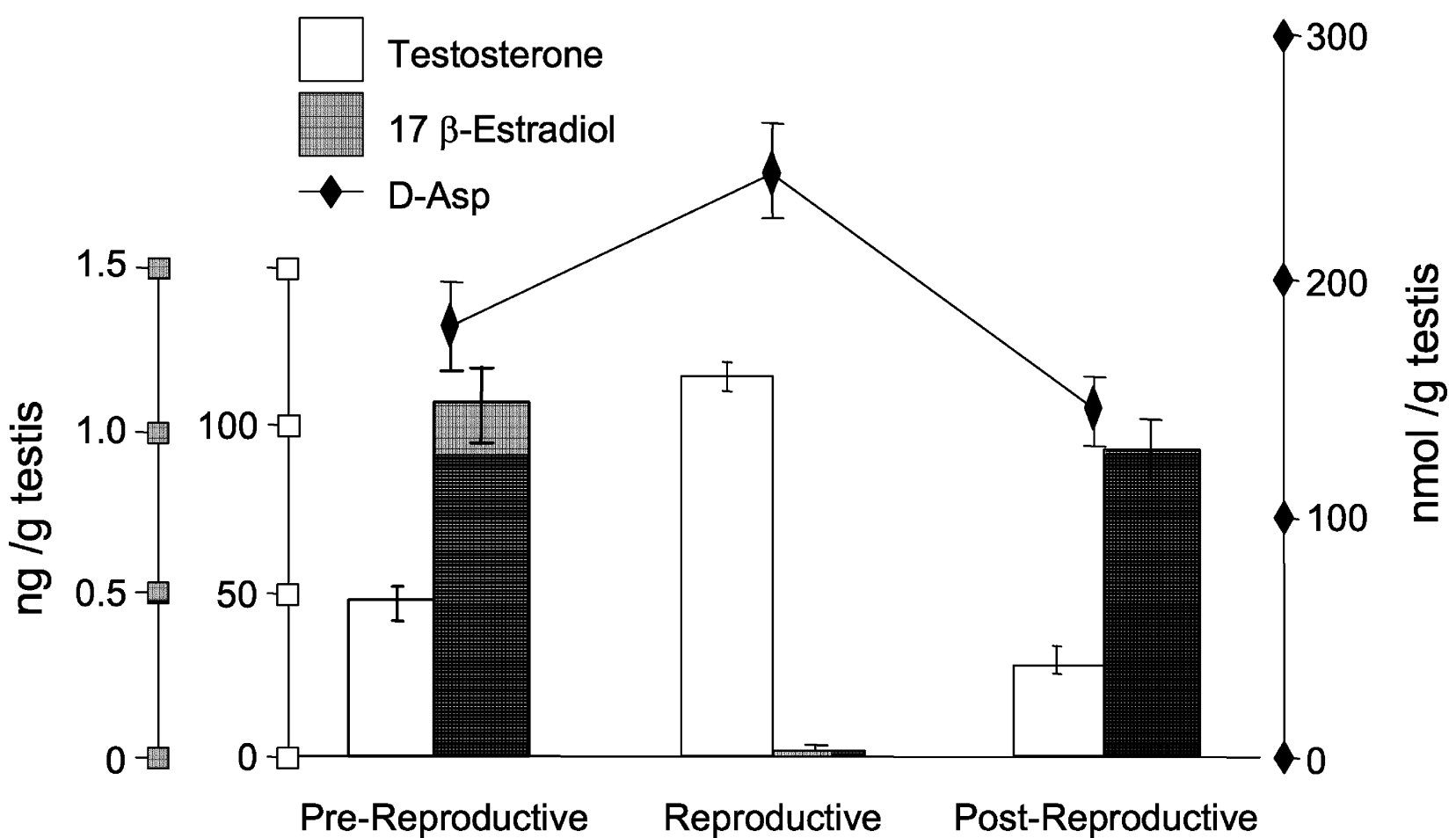

Figure 1 Concentrations of D-Asp and titers of testosterone and $17 \beta$-estradiol in the testis of $R$. esculenta during the reproductive cycle. Each point represents the mean value \pm S.D. from five individual frogs.

running LUCIA ScMeas software (Laboratory Image Ltd, Praha, Czech Republic). The measurements were taken by computer mouse-directed tracing of the digitized images on a video display screen.

\section{Statistical analysis}

Data were compared by analysis of variance followed by Duncan's test for multigroup comparison and Student's $t$-test for between-group comparison. All data were expressed as mean \pm standard deviations. The level of significance was taken at $P<0 \cdot 01$ and $P<0 \cdot 05$.

\section{Results}

Figure 1 reports the profiles of D-Asp and sex steroid (testosterone and $17 \beta$-estradiol) content in the testis of $R$. esculenta. This amphibian is a seasonal breeder, mating and egg deposition usually occurring in early spring; therefore, animals were examined during the three main phases of their reproductive cycle, that is, pre-reproductive (October), reproductive (February-March) and postreproductive (July). As indicated in the graph, in all three phases of the cycle, substantial amounts of free D-Asp were found in testicular extracts $(140-236 \mathrm{nmol} / \mathrm{g}$ of fresh tissue). This amino acid level showed significant variations, depending on the reproductive cycle phase, reaching its highest content in the testis of animals engaged in sexual activity. The D-Asp level in the testis positively matched the testosterone level, since this hormone titer was also higher during the reproductive phase of the cycle in the testis (Fig. 1) and in the blood (not shown). $17 \beta$-estradiol was present in the testis, although at lower concentration, in the pre-reproductive and postreproductive phases, but it was almost undetectable in the reproductive phase (Fig. 1).

The endogenous presence of $\mathrm{D}-\mathrm{Asp}$ in $R$. esculenta suggested that this amino acid came from L-Asp through the activity of racemase, an enzyme which converts L-Asp to D-Asp, as demonstrated in rats (Wolosker et al. 2000). For this study L-Asp was incubated with a homogenate of testis or liver (two tissues where we expected to find racemase), and the formation of D-Asp was determined. The results from this investigation have demonstrated that such racemase activity does occur in the liver and testis of $R$. esculenta (Table 1). We found that in both tissues the racemase activity was present, with the maximum at $\mathrm{pH} 7 \cdot 0$, while at $\mathrm{pH} 6 \cdot 0$ and $8 \cdot 0$ the activity was about $80 \%$ of $\mathrm{pH} 7 \cdot 0$. The greatest D-Asp biosynthesis was found in liver, with about $50 \mathrm{U} / \mathrm{g}$ tissue. However, this value remained unchanged through the different periods of the cycle (Table 1). In the testis, the activity of this enzyme was less concentrated than in the liver; however, in this 
Table 1 Aspartate racemase activity during reproductive cycle (enzymatic unity/g tissue)

\begin{tabular}{|c|c|c|c|}
\hline & Pre-reproductive & Reproductive & Post-reproductive \\
\hline \multicolumn{4}{|l|}{ Tissue } \\
\hline \multicolumn{4}{|c|}{ Testis incubated with } \\
\hline L-Asp & $9 \cdot 1 \pm 2 \cdot 0^{*}$ & $25 \cdot 3 \pm 3 \cdot 9^{*}$ & $5 \cdot 5 \pm 1 \cdot 2^{*}$ \\
\hline L-Glu & $2 \cdot 2 \pm 0 \cdot 3$ & $2 \cdot 6 \pm 0 \cdot 4$ & $2 \cdot 1 \pm 0 \cdot 3$ \\
\hline L-Ala & $2 \cdot 5 \pm 0 \cdot 4$ & $2 \cdot 7 \pm 0 \cdot 5$ & $1 \cdot 9 \pm 0 \cdot 2$ \\
\hline Control & $2 \cdot 3 \pm 0 \cdot 3$ & $2 \cdot 4 \pm 0 \cdot 3$ & $1 \cdot 8 \pm 0 \cdot 2$ \\
\hline \multicolumn{4}{|c|}{ Liver incubated with } \\
\hline L-Asp & $48 \cdot 5 \pm 5 \cdot 2^{*}$ & $51 \cdot 4 \pm 4 \cdot 9^{*}$ & $50 \cdot 2 \pm 4 \cdot 8^{*}$ \\
\hline L-Glu & $4 \cdot 2 \pm 0 \cdot 9$ & $4 \cdot 8 \pm 1 \cdot 0$ & $3 \cdot 1 \pm 0 \cdot 7$ \\
\hline L-Ala & $3 \cdot 7 \pm 0 \cdot 5$ & $4 \cdot 0 \pm 0 \cdot 9$ & $2 \cdot 7 \pm 0 \cdot 4$ \\
\hline Control & $3 \cdot 3 \pm 0 \cdot 7$ & $4 \cdot 5 \pm 1 \cdot 1$ & $2 \cdot 8 \pm 0 \cdot 6$ \\
\hline
\end{tabular}

The results represent mean \pm s.D. obtained from five individual frogs. One enzymatic unity was defined as the amount of enzyme able to develop $1 \mathrm{nmol}$ of D-Asp from L-Asp under the assay conditions (see Materials and Methods). ${ }^{*} P<0 \cdot 01$ vs control.

tissue, there is a variation of racemase concentration in different periods of the year. High activity was observed during the reproductive cycle, where the values were found to be $25 \cdot 3 \pm 3 \cdot 9 \mathrm{U} / \mathrm{g}$, as opposed to $9 \cdot 1 \pm 2 \cdot 0$ in the pre-reproductive period, and $5.5 \pm 1 \cdot 2$ in the postreproductive period (Table $1, P<0 \cdot 01$ ). To verify the racemase activity in either testis or liver, the tissues were also incubated with other L-amino acids (L-Glu or L-Ala), but there were no differences from the control (tissues incubated with $\mathrm{NaCl}$ instead of amino acids), meaning that the only substrate for the $\mathrm{D}$-aspartate racemase was L-Asp (Table 1).

Figure 2 reports the profile of D-Asp uptake by the gonad (A) together with testosterone levels in the gonad (B) and in the plasma (C) of frogs caught in the postreproductive period and injected with a single dose of exogenous D-Asp $(2 \cdot 0 \mu \mathrm{mol} / \mathrm{g}$ body weight $)$ or saline solution alone (control). As shown in Fig. 2, after D-Asp was injected into the animal, the D-Asp level rapidly rose in the testis, reaching its highest concentration $6 \mathrm{~h}$ after the administration. Thereafter, it progressively decreased to basal values within $24 \mathrm{~h}$. At least during the first $3 \mathrm{~h}$, the D-Asp increase in the gonad was coupled to a significant increase of testosterone in the gonad. Thereafter, the levels returned to baseline in $6 \mathrm{~h}$. The effects of D-Asp on the concentration of testosterone in the plasma were similar to those observed in the testis (Fig. 2C).

To determine the effects of D-Asp and other amino acids on testosterone synthesis and release, we carried out a long-term experiment in which different groups of post-reproductive male frogs were administered $2 \cdot 0 \mu \mathrm{mol} / \mathrm{g}$ body weight of amino acids on alternate days for 2 weeks. The results obtained from this experiment have indicated that in the testis D-Asp induces a very significant increase of testosterone (from basal value of about $76 \mathrm{ng} / \mathrm{g}$ testis, D-Asp increases testosterone to about $175 \mathrm{ng} / \mathrm{g}$; this increase was significant at $P<0 \cdot 01)$. Other
D- and L-amino acids tested (L-Glu, D-Glu and L-Asp) did not induce significant synthesis and release of testosterone (Fig. 3A), meaning that only D-Asp possesses such activity. A similar effect was also observed in the blood (Fig. 3B).

Figure 4 reports the results of a long-term experiment in which D-Asp was administered to frogs caught in the three studied phases of the sexual cycle. It was observed that $\mathrm{D}$-Asp induced a testosterone increase in the gonad during the pre-reproductive and post-reproductive period, but not during the reproductive period when the testosterone content of the gonad was at its highest seasonal level. Conversely, the D-Asp treatment was followed by a decrease of $17 \beta$-estradiol level in the pre-reproductive and post-reproductive frog testis and by an increase of this hormone titer in reproductive frogs. Moreover, the $17 \beta-$ estradiol level decreased whenever testosterone increased.

In other experiments, the testis and thumb pad of frogs were utilized for morphologic studies (Figs 5 and 6 respectively). As shown in Fig. 5, it was only in the D-Asp-treated animals (B) that an increase of seminiferous ampoule area with respect to the control (A) occurred (27 $852 \pm 6205$ vs $\left.62237 \pm 6970 \mu \mathrm{m}^{2}, P<0 \cdot 01\right)$. In the seminiferous ampoules of treated animals, we found a higher number of spermatids and spermatocytes (Fig. 5B). Immunocytochemistry analysis revealed the presence of PCNA protein in the $R$. esculenta testis (Fig. $5 \mathrm{C}$ and D). The immunopositivity was found in the mitotic germinal epithelium and was localized in the cytoplasm and nucleus of I and II spermatogonia, and in the nuclei of I spermatocytes of both control (Fig. 5C) and D-Asp-treated (Fig. 5D) animals. Moreover, the testes of frogs treated with D-Asp showed a greater intensity of immunoreaction (Fig. 5D).

In addition, the thumb pads of D-Asp-treated frogs was stimulated, since their epithelial lining was thicker (Fig. 6B) than in the controls (Fig. 6A), and their glands had a significantly wider area than those of control frogs $\left(4370 \pm 827\right.$ vs $7063 \pm 885 \mu \mathrm{m}^{2}, P<0 \cdot 01$, Fig. $6 \mathrm{D}$ vs 


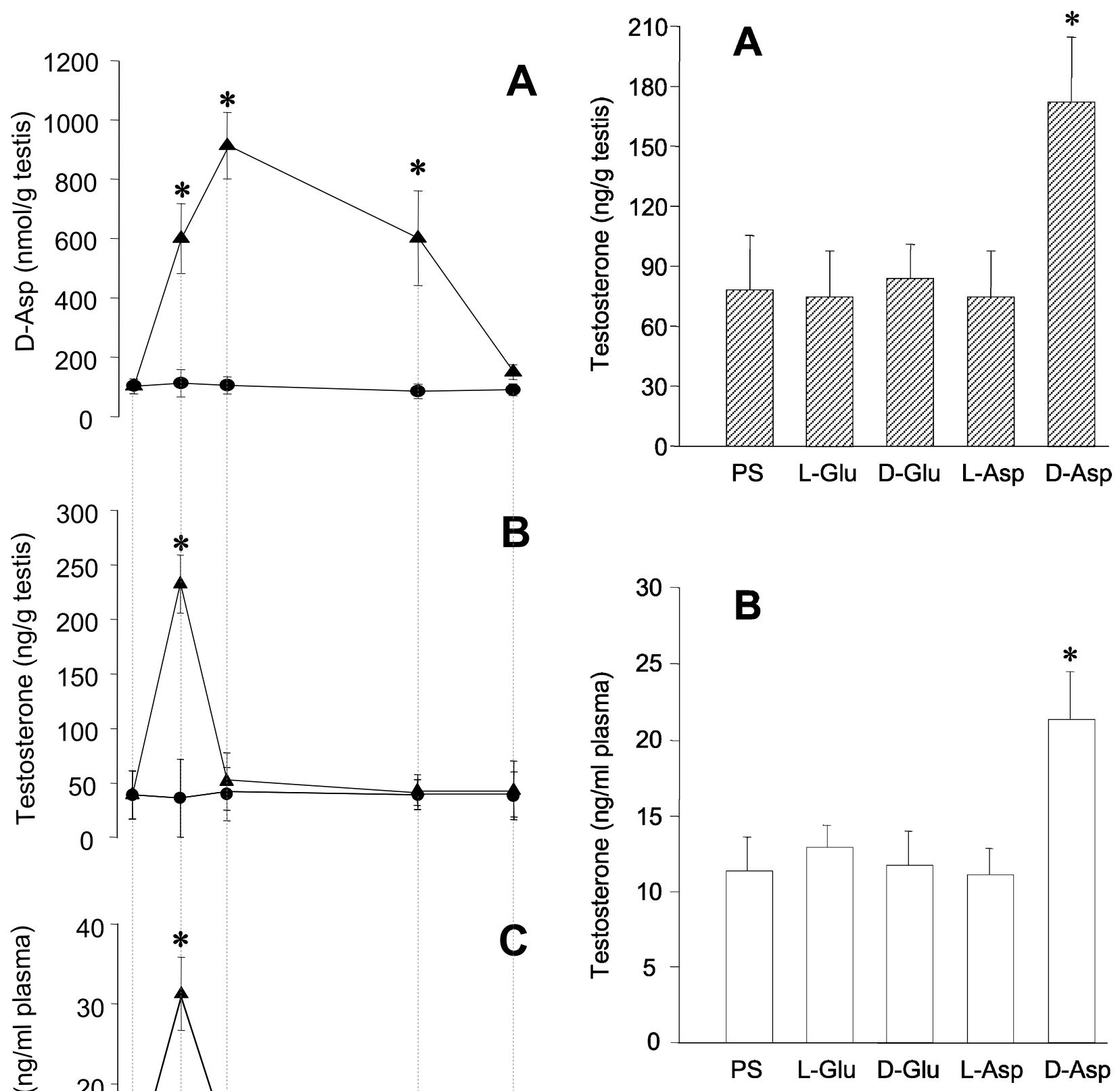

Figure 3 Effect of administering different amino acids on testis (A) and plasma (B) concentrations of testosterone in $R$. esculenta. PS: physiological saline, L-Glu: L-glutamate, D-Glu: D-glutamate, L-Asp: L-aspartate, D-Asp: D-aspartate. Each value represents mean \pm S.D. of five determinations; ${ }^{*} P<0 \cdot 01$.

6C). In each gland, the secretory cells were taller $(81.65 \pm 7.84$ vs $133.33 \pm 15.98 \mu \mathrm{m}, P<0 \cdot 01$, Fig. $6 \mathrm{D})$ and more numerous $(35.50 \pm 5.93$ vs $61.50 \pm 8.26$ cells for gland section, $P<0 \cdot 01$, Fig. 6D) than in controls (Fig. 6C). PAS reaction for mucosubstances was strongly positive in the glands of D-Asp-treated frogs (Fig. 6F) compared with the control group (Fig. 6E). 
Testosterone

Testosterone after D-Asp treatment

\section{$17 \beta$-Estradiol}

\section{$17 \beta$-Estradiol after \\ D-Asp treatment}

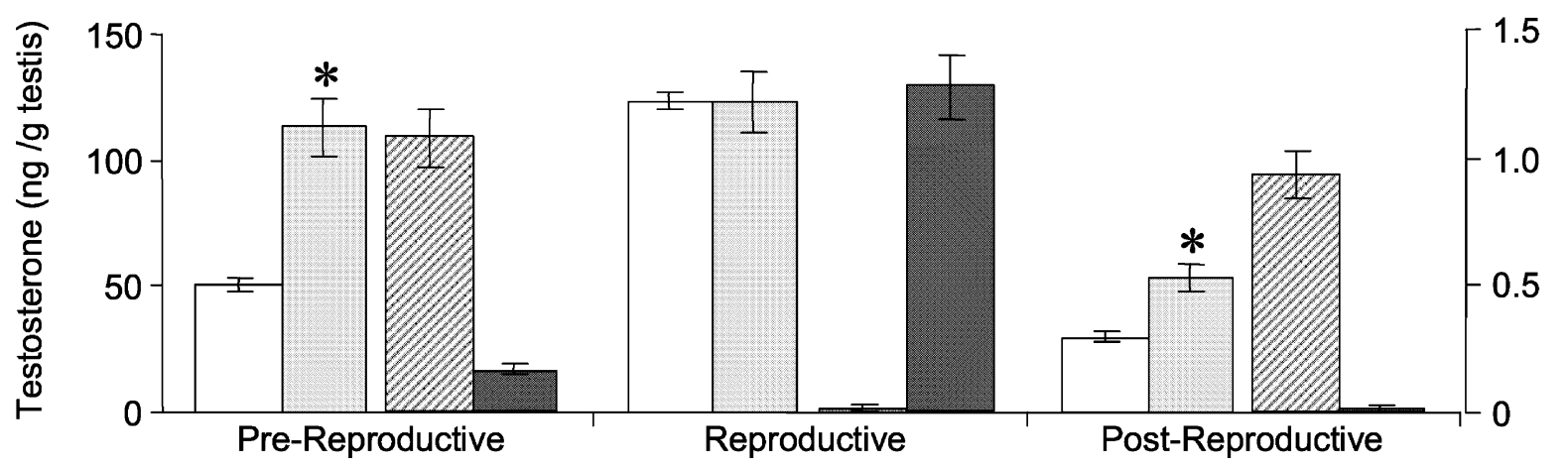

Figure $4 \mathrm{Effect}$ of D-Asp $(2 \cdot 0 \mu \mathrm{mol} / \mathrm{g}$ body weight) on testis concentrations of testosterone and $17 \alpha$-estradiol in $R$. esculenta, during the reproductive cycle. Each value represents mean \pm S.D. of five determinations; ${ }^{*} P<0 \cdot 01$.

\section{Discussion}

The aim of this study was to ascertain whether D-Aspdependent testosterone production, well documented in the mammalian testis, is a specific feature of this vertebrate male gonad or a physiological property of D-Asp that is widespread in other vertebrates. Most studies carried out on this peculiar D-Asp function have been devoted to mammals, especially the rat. In these animals, D-Asp facilitates testosterone synthesis through either indirect or direct actions. The first involves the hypothalamuspituitary-gonad axis (D'Aniello et al. 2000a), whereas the second operates in Leydig cell machinery assigned to sex steroid synthesis (Nagata et al. 1999a,b). The present study results on the male green frog, $R$. esculenta, confirm a vertebrate-wide D-Asp function in steroidogenesis. This finding is supported by the relationships among the concentrations of D-Asp, testosterone and $17 \beta$-estradiol that were observed during the reproductive cycle of the frog and under experimental conditions. Moreover, D-Asp can positively affect the maturation of spermatogenesis and the development of a typical secondary sexual characteristic (SSC) in this species, the thumb pad (Di Fiore et al. 2002).

Substantial amounts of D-Asp are endogenously contained in the frog testis, and levels of this amino acid change throughout the sexual reproductive cycle, positively matching the testosterone levels in the gonad and blood. Moreover, in short-term experiments on male frogs, where a single dose of $\mathrm{D}$-Asp was injected, this amino acid was rapidly taken up by the gonad, and its rise was coupled with a significant increase of testosterone concentration in the testis. The increase in D-Asp-treated males occurred within $3 \mathrm{~h}$ of the amino acid injection: at that time, the concentration of $\mathrm{D}-\mathrm{Asp}$ was about $600 \mathrm{nmol} / \mathrm{g}$ tissue. This means that the quantity was already enough to induce the maximum testosterone release, and further amounts of D-Asp were ineffective. The effect of D-Asp on testosterone was reversible; in fact, thereafter $(3-24 \mathrm{~h})$ the hormone concentration was rapidly restored until baseline. These D-Asp effects appear to be specific since they do not occur in frogs treated with other D- or L-amino acids, that is, the L-form of aspartate and the D- and L-forms of glutamate, the other amino acids with known excitatory effects on neurosecretion.

In long-term experiments, D-Asp induced an increase of testosterone production when injected into prereproductive and post-reproductive males, confirming the relationships between testosterone and D-Asp observed during the reproductive cycle: a greater D-Asp content in the testis coincides with the peaks of testosterone content both in the testis and in the plasma. These findings suggest that, as in other vertebrates, testosterone production by the testis is also under the control of endogenous D-Asp. In our study, however, the D-Asp-dependent testosterone testicular increase was not noticed in reproductive D-Asptreated frogs, probably because, during this sexual cycle phase, the high endogenous D-Asp content helps keep the testosterone production at its maximum level.

Some investigators have obtained evidence of D-Asp synthesis. Long et al. (1998, 2002) demonstrated the intracellular synthesis of D-Asp in mammalian cells for the first time, and Wolosker et al. (2000) reported an aspartate racemase which is physiologically responsible for the formation of D-Asp in rat. In the cerebral ganglion of the protochordate Ciona intestinalis, it has recently been demonstrated that D-Asp is synthesized from L-Asp by an aspartate racemase (D'Aniello et al. 2003). Here we demonstrate that an aspartate racemase which specifically generates D-Asp acting only on L-Asp as substrate is 

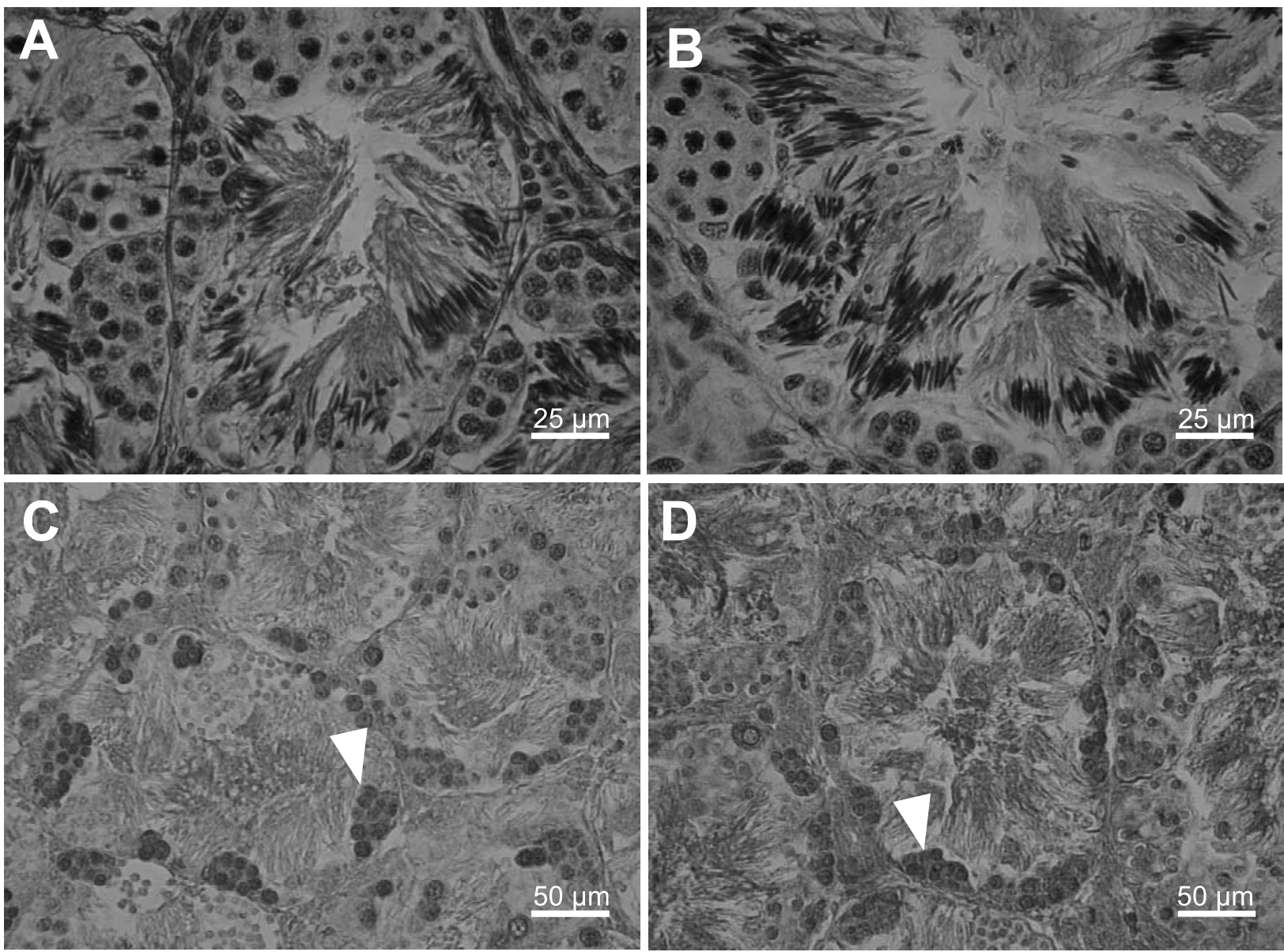

Figure 5 Histologic sections of control (A) and D-Asp-treated (B) testis of R. esculenta. Seminiferous ampoules of treated animals showed a higher number of spermatids and sperm than in the control (HE $\times 400)$. Immunocytochemistry for PCNA in the testis of $R$. esculenta treated with D-Asp (D) showed major intensity immunopositive spermatogonial proliferation (arrowhead), with respect to the controls (C) ( $\times 200)$. Scale bars: A and B, $25 \mu \mathrm{m} ; \mathrm{C}$ and D, $50 \mu \mathrm{m}$.

present in $R$. esculenta. This enzyme occurs in both liver and testes. In the liver, D-Asp synthesis remains unchanged throughout the entire reproductive cycle. By contrast, in the testes, there are significant changes, and racemase activity peaks in the reproductive period when testosterone levels are the highest during spermatogenesis and reproduction. Thus, we are inclined to argue that at least a part of the D-Asp formed in the testis during the reproductive phase can contribute to the formation of more testosterone.

The highest levels of circulating testosterone during the reproductive phase are beneficial for spermatogenesis and the development and maturation of the thumb pad, a typical androgen-dependent target organ (Delrio et al. 1980, Varriale \& Serino 1994, Di Fiore et al. 2002). As for spermatogenesis, the D-Asp treatment causes an increase of seminiferous ampulae area and their spermatid and sperm contents. The spermatogenesis induction is supported by the PCNA immunopositivity in the cytoplasm and nucleus of I and II spermatogonia and in the nucleus of early stage I spermatocytes. Moreover, thumb pad histologic and histochemical analysis of D-Asp-treated frogs showed that their epithelial lining was stimulated and their secretory glands presented an accumulation of PASpositive material in the cell gland, compared with the control group. The stimulation of thumb pad morphology and glandular activity suggests that enough of this hormone has reached this organ, which is a known testosteronedependent SSC in this amphibian species (Varriale \& Serino 1994, Delrio et al. 1980, Di Fiore et al. 2002).

In mammals, recent studies have clarified the testicular action mechanism of D-Asp in testosterone production. D-Asp upregulates StAR gene expression in the Leydig cells, entailing an increase of StAR protein synthesis (Nagata et al. 1999b). This protein, in turn, is assumed to facilitate, by a yet undefined mechanism, the translocation of cholesterol to the inner mitochondrial membrane, a rate-limiting process in steroid synthesis (Nagata et al. 

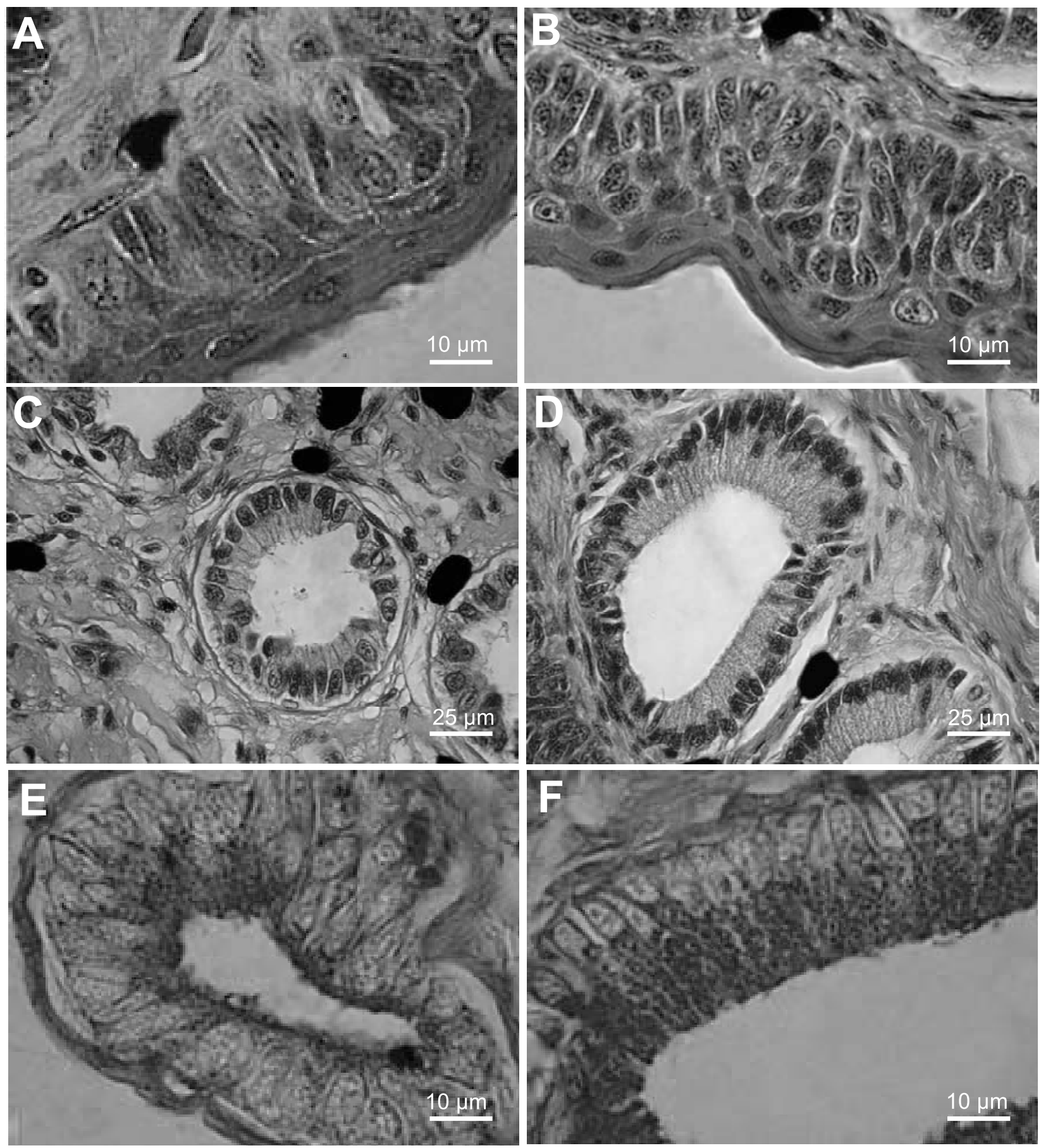

Figure 6 Paraffin sections of thumb pads from R. esculenta. (A) Epithelial lining of a control frog (HE $\times 1000)$. (B) In D-Asp-treated frogs, the epithelial lining was thicker than in the controls $(\mathrm{HE} \times 1000)$. (C) One seromucous gland of thumb pad from a control frog $(\mathrm{HE} \times 400)$. (D) The glandular epithelium of the D-Asp-treated frogs showed secretory cells taller and more numerous than in controls $(\mathrm{HE} \times 400)$. (E) The glandular epithelium from a control showed the apical cytoplasm positive to PAS reaction (PAS $\times 1000)$. (F) The glandular epithelium from D-Asp-treated frogs showed cytoplasm strongly filled by PAS-positive mucosubstances in the secretory glands (PAS $\times 1000$ ). Scale bars: A, B, E and F, $10 \mu \mathrm{m}$; C and D, $25 \mu \mathrm{m}$. 
1999a). Although a similar D-Asp mechanism of action is also likely to operate in frog testis, no data are available to sustain such a proposition.

The D-Asp-dependent testosterone increases could also follow D-Asp intervention in testicular aromatase activity. In fact, during the sexual cycle phases of $R$. esculenta males, $17 \beta$-estradiol testicular levels inversely match those of either D-Asp or testosterone. Chronic D-Asp injection when testosterone levels are relatively low in gonads and blood, that is, in pre- and post-reproductive captive frogs, induces a testosterone increase and a clear $17 \beta$-estradiol decrease. In contrast, when this treatment is carried out on reproductive frogs, it causes an increase of $17 \beta$-estradiol level. In the reproductive period, when the testosterone production is kept at its maximum by endogenous high D-Asp content, the additional D-Asp administration causes a stimulation of aromatase activity, which prevents any excess testosterone production.

The D-Asp effect on aromatase activity in $R$. esculenta males is consistent with the observations carried out on the lizard Podarcis s. sicula (Assisi et al. 2001). In the ovary of this species, a positive correlation between D-Asp and $17 \beta$-estradiol content has been shown. The results of in vivo and in vitro experiments, moreover, indicated that in these females D-Asp enhances the ovarian aromatase activity, favoring testosterone conversion to $17 \beta$-estradiol. This mechanism provides a high estrogen supply, which is of great physiological value since it sustains vitellogenin synthesis by the liver during the pre-reproductive phase of the cycle. Furthermore, in $R$. esculenta females, it was demonstrated that $\mathrm{D}$-Asp induced a decrease of testosterone and a concomitant increase of $17 \beta$-estradiol. Although there are no studies of D-Asp effects on estrogen production by mammalian female gonads, these observations suggest that D-Asp influx on the gonadal aromatase activity may depend on the sex. At least in R. esculenta, the amino acid could facilitate testosterone production in males and $17 \beta$-estradiol in females by inhibiting and stimulating local activity respectively.

In conclusion, our results provide new insight into the biologic function of D-Asp in amphibian male steroidogenesis. Future investigations should address the precise mechanism of action of this $\mathrm{D}$-amino acid. In view of the extensive research literature on D-Asp and its dose response in the regulation of various hormones (Di Fiore et al. 1998, D'Aniello et al. 1998b, Nagata et al. 1999a, D'Aniello et al. 2000a,b, Wang et al. 2000, 2002, Assisi et al. 2001), we propose that D-Asp has an important role in the modulation of some processes connected with reproduction in vertebrates.

\section{Acknowledgement}

This work was supported by MIUR grant (cofin PRIN 2001, prot. 2001058119).

\section{References}

Assisi L, Botte V, D’Aniello A \& Di Fiore MM 2001 Enhancement of aromatase activity by D-aspartic acid in the ovary of the lizard Podarcis s. sicula. Reproduction 121 803-808.

Chieffi P, Franco R, Fulgione D \& Staibano S 2000 PCNA in the testis of the frog, Rana esculenta: a molecular marker of the mitotic testicular epithelium proliferation. General and Comparative Endocrinology 119 11-16.

D'Aniello A \& Giuditta A 1977 Identification of D-aspartic acid in the brain of Octopus vulgaris Lam. Journal of Neurochemistry 29 1053-1057.

D’Aniello A \& Giuditta A 1978 Presence of D-aspartate in squid axoplasma and other regions of the cephalopod nervous system. Journal of Neurochemistry 31 1107-1108.

D'Aniello A, Nardi G, Vetere A \& Ferguson GP 1993a Occurrence of free D-aspartic acid in the circumoesophageal ganglia of Aplysia fasciata. Life Sciences $\mathbf{5 2}$ 733-736.

D'Aniello A, Vetere A \& Petrucelli L 1993b Further study on the specificity of D-amino acid oxidase and of D-aspartate oxidase and time course for complete oxidation of $\mathrm{D}$-amino acids. Comparative Biochemistry and Physiology 105B 731-734.

D’Aniello A, Di Fiore MM \& Fisher G 1998a Occurrence of D-aspartic acid in animal tissues and its role in the nervous and endocrine systems. Trends in Comparative Biochemistry and Physiology 4 1-21.

D'Aniello A, Di Fiore MM, D'Aniello G, Colin FE, Lewis G \& Setchell BP 19986 Secretion of D-aspartic acid by the rat testis and its role in endocrinology of the testis and spermatogenesis. FEBS Letters 436 23-27.

D'Aniello A, Di Fiore MM, Fisher GH, Milone A, Seleni A, D'Aniello S, Perna AF \& Ingrosso D 2000a Occurrence of D-aspartic acid and $N$-methyl-D-aspartic acid in rat neuroendocrine tissues and their role in the modulation of luteinizing hormone and growth hormone release. FASEB Journal 14 699-714.

D'Aniello G, Tolino A, D'Aniello A, Errico F, Fisher GH \& Di Fiore MM $2000 b$ The role of D-aspartic acid and N-methyl-D-aspartic acid in the regulation of prolactin release. Endocrinology 141 3862-3870.

D'Aniello A, Spinelli P, De Simone A, D'Aniello S, Branno M, Aniello F, Fisher GH, Di Fiore MM \& Rastogi RK 2003 Occurrence and neuroendocrine role of D-aspartic acid and $N$-methyl-D-aspartic acid in Ciona intestinalis. FEBS Letters $\mathbf{5 5 2}$ 193-198.

Delrio G, Citarella F \& d'Istria M 1980 Androgen receptor in the thumb pad of Rana esculenta: dynamic aspects. Journal of Endocrinology 85 279-282.

Di Fiore MM, Assisi L, Botte V \& D’Aniello A 1998 D-Aspartic acid is implicated in the control of testosterone production by the vertebrate gonad. Studies on the female green frog Rana esculenta. Journal of Endocrinology 157 199-207.

Di Fiore MM, Chieffi Baccari G, Rastogi RK, Di Meglio M, Pinelli C \& Iela L 2002 Hormonal regulation of secondary sexual characters. In Biology of Amphibia, vol. VI (Endocrinology). Ed. W Heatwole. Sydney: Surrey Beatty.

Dunlop DS, Neidle A, McHale D, Dunlop DM \& Lajtha A 1986 The presence of free D-aspartic acid in rodents and man. Biochemical and Biophysical Research Communications 141 27-32.

Fisher GH, D'Aniello A, Vetere A, Padula L, Cusano GP \& Man EH 1991 Free D-aspartate and D-alanine in normal and Alzheimer brain. Brain Research Bulletin 26 983-985.

Hashimoto A \& Oka T 1997 Free D-aspartate and D-serine in the mammalian brain and periphery. Progress in Neurobiology 52 325-353.

Hashimoto A, Nishikawa T, Oka T, Hayashi T \& Takahashi K 1993 Widespread distribution of free $\mathrm{D}$-aspartate in rat periphery. FEBS Letters 331 4-8.

Hsu SM, Raine L \& Fanger H 1981 Use of avidin-biotinperoxidase complex $(\mathrm{ABC})$ in immunoperoxidase techniques: a comparison 
between $\mathrm{ABC}$ and unlabeled antibody (PAP) procedures. Journal of Histochemistry and Cytochemistry 29 577-580.

Imai K, Fukushima T, Hagiwara K \& Santa T 1995 Occurrence of D-aspartic acid in rat brain pineal gland. Biomedical Chromatography 9 106-109.

Ishio S, Yamada H, Hayashi M, Yatsushiro S, Noumi T, Yamaguchi A \& Moriyama Y 1998 D-Aspartate modulates melatonin synthesis in rat pinealocytes. Neuroscience Letters 249 143-146.

Lee JA, Homma H, Sakai K, Fukushima T, Santa T, Tashiro K, Iwatsubo T, Yoshikawa M \& Imai K 1997 Immunohistochemical localization of $\mathrm{D}$-aspartate in rat pineal gland. Biochemical and Biophysical Research Communications 231 505-508.

Lee JA, Long Z, Nimura N, Iwatsubo T, Imai K \& Homma H 2001 Localization, transport, and uptake of $\mathrm{D}$-aspartate in the rat adrenal and pituitary glands. Archives of Biochemistry and Biophysics $\mathbf{3 8 5}$ 242-249.

Long Z, Homma H, Lee JA, Fukushima T, Santa T, Iwatsubo T, Yamada R \& Imai K 1998 Biosynthesis of D-aspartate in mammalian cells. FEBS Letters 434 231-235.

Long Z, Sekine M, Adachi M, Furuchi T, Imai K, Nimura N \& Homma H 2002 Cell density inversely regulates L- and D-aspartate levels in rat pheochromocytoma MPT1 cells. Archives of Biochemistry and Biophysics 404 92-97.

Mazzi V 1977 Manuale di tecniche istologiche e istochimiche. Ed Mazzi V. Padova: Piccin.

Nagata Y, Homma H, Lee JA \& Imai K 1999a D-Aspartate stimulation of testosterone synthesis in rat Leydig cells. FEBS Letters 444 160-164.

Nagata Y, Homma H, Matsumoto M \& Imai K 1999 b Stimulation of steroidogenic acute regulatory protein (StAR) gene expression by D-aspartate in rat Leydig cells. FEBS Letters 454 317-320.

Negri A, Tedeschi G, Ceciliani F \& Ronchi S 1999 Purification of beef kidney D-aspartate oxidase overexpressed in Escherichia coli and characterization of its redox potentials and oxidative activity towards agonists and antagonists of excitatory amino acid receptors. Biochimica et Biophysica Acta 1431 212-222.
Sakai K, Homma H, Lee JA, Fukushima T, Santa T, Tashiro K, Iwatsubo T \& Imai K 1998 Localization of D-aspartic acid in elongate spermatids in rat testis. Archives of Biochemistry and Biophysics 351 96-105.

Schell MJ, Cooper OB \& Snyder SH 1997 D-Aspartate localizations imply neuronal and neuroendocrine roles. PNAS 94 2013-2018.

Takigawa Y, Homma H, Lee JA, Fukushima T, Santa S, Iwatsubo T \& Imai K 1998 D-Aspartate uptake into cultured rat pinealocytes and the concomitant effect on L-aspartate levels and melatonin secretion. Biochemical and Biophysical Research Communications 248 641-647.

Tedeschi P, Negri A, Ceciliani F, Ronchi S, Vetere A, D'Aniello G \& D'Aniello A 1994 Properties of the flavoenzyme D-aspartate oxidase from Octopus vulgaris. Biochimica et Biophysica Acta 1207 217-222.

Varriale B \& Serino I 1994 The androgen receptor mRNA is up-regulated by testosterone in both the Harderian gland and thumb pad of the frog, Rana esculenta. Journal of Steroid Biochemistry and Molecular Biology 51 259-265.

Wang H, Wolosker H, Pevsner J, Snyder SH \& Selkoe DJ 2000 Regulation of rat magnocellular neurosecretory system by D-aspartate: evidence for biological role(s) of a naturally occurring free D-amino acid in mammals. Journal of Endocrinology 167 247-252.

Wang H, Wolosker H, Morris JF, Pevsner J, Snyder SH \& Selkoe DJ 2002 Naturally occurring free D-aspartate is a nuclear component of cells in the mammalian hypothalamo-neurohypophyseal-system. Neuroscience 109 1-4.

Wolosker H, D’Aniello A \& Snyder SH 2000 D-Aspartate disposition in neuronal and endocrine tissues: ontogeny, biosynthesis and release. Neuroscience 100 183-189.

Received 7 April 2004

Accepted 19 April 2004 